\title{
Evidence That L-Dopa-Induced Rotational Behavior Is Dependent on Both Striatal and Nigral Mechanisms
}

\author{
G. S. Robertsona and H. A. Robertson \\ Department of Pharmacology, Faculty of Medicine, Dalhousie University, Halifax, Nova Scotia B3H 4H7, Canada
}

Parkinson's disease results from the death of the dopaminecontaining neurons in the substantia nigra pars compacta (SNC). This is accompanied by a loss of dopamine in brain regions, such as the corpus striatum, which receives input from dopaminergic neurons in the substantia nigra (SN). Since the corpus striatum is the primary target for these dopaminergic neurons, it has long been thought that the corpus striatum is the principal region affected. It was, therefore, natural to assume that replenishing dopamine in the striatum might be an effective treatment for Parkinson's disease. In fact, the dopamine precursor L-dihydroxyphenylalanine (L-dopa), the current drug of choice for treatment of Parkinson's disease, is believed to exert its therapeutic effect by replenishing dopamine levels in the corpus striatum via enzymatic decarboxylation within the synaptic terminals of surviving nigrostriatal neurons (Hornykiewicz, 1974). However, dopamine is also synthesized, stored, and released from the dendrites of SNC neurons that arborize in the substantia nigra pars reticulata (SNR) (Cheramy et al., 1981). Using a classic animal model for Parkinson's disease (rats with a unilateral 6-hydroxydopamine lesion of the SN), we show that L-dopa is also converted to dopamine in significant amounts within the 6-OHDA-lesioned SN. Furthermore, in contrast to the situation in the striatum where dopamine levels are only elevated for a short time, dopamine levels in the SN remain elevated until the behavioral effects of L-dopa have subsided. This elevation of nigral dopamine levels produces rotation that can be blocked by injecting a selective D1 dopamine receptor antagonist (SCH 23390, $2 \mu \mathrm{g}$ in $1 \mu \mathrm{l}$ ) directly into the SN pars reticulata. Infusion of SCH 23390 into the ipsilateral striatum produced only a modest reduction in L-dopa-induced circling behavior. These results suggest that D1 dopamine receptors in the SN may be at least as important as D1 dopamine receptors in the striatum as a site for the effects of L-dopa. This may have important implications for the therapy of Parkinson's disease.

\footnotetext{
Received December 1, 1988; revised March 14, 1989; accepted March 15, 1989.

We are grateful to Marc Peterson for technical assistance, to $\mathrm{M}$. Wilkinson and A. Fine for comments on the manuscript, and to Merck Frosst Canada Inc. for carbidopa, and the Schering Corporation for SCH 23390. G.S.R. holds a predoctoral studentship from the Medical Research Council for Canada. This work was supported by grants to H.A.R. from Sandoz (Canada) Inc. and the Parkinson Foundation of Canada.

Correspondence should be addressed to Dr. H. A. Robertson at the above address.

a Present address: Kinsman Laboratory, Department of Neurological Science, University of British Columbia, Vancouver, British Columbia V6T 1 W5.

Copyright (C) 1989 Society for Neuroscience $0270-6474 / 89 / 093326-06 \$ 02.00 / 0$
}

The localization of dopamine in the dendritic network arising from cell bodies of the dopaminergic neurons in the substantia nigra (SN) was first reported by Björklund and Lindvall (1975). Since then, considerable evidence has accumulated confirming that the dopaminergic neurons of the substantia nigra pars compacta (SNC) are unique in that dopamine can be synthesized, stored, and released not only from the terminal fields that innervate the striatum but also from the dendritic network that arborizes in the substantia nigra pars reticulata (SNR) (Geffen et al., 1976; Cheramy et al., 1981; Gauchy et al., 1987).

Dopamine released from the dendrites of the SNC neurons has been proposed to act in both autocrine and paracrine fashion. In the case of the former, dendritic dopamine has been suggested to modulate SNC activity by stimulating D2-type dopamine receptors located on the soma and dendrites of SNC neurons, i.e., autoreceptors (Nissbrant et al., 1985). The autorcceptor hypothesis was first proposed by Bunney and Aghajanian (1973) on the basis of a reduction in firing rate of SNC neurons after microiontophoretic application of dopamine, which was reversed by neuroleptics. However, the high density of D1type dopamine receptors localized on the terminals of striatal efferents that innervate the SNR suggests that dendritically released dopamine also may have a paracrine action (Robertson and Robertson, 1987a, b). This notion is supported by the finding that dopamine selectivity increases ${ }^{3} \mathrm{H}-\mathrm{GABA}$ release from tissue slices of rat SN (Reubi et al., 1977). It seems likely, therefore, that dendritically released dopamine may increase GABA release in the $\mathrm{SN}$ by activating $\mathrm{D} 1$ dopamine receptors on the terminals of striatonigral efferents (Robertson and Robertson, $1987 \mathrm{a}, \mathrm{b})$. Interestingly, intranigral administration of dopamine increases spontaneous locomotion in rats, suggesting that dendritic release of dopamine may have a functional role in the SN (Jackson and Kelly, 1983). Consequently, activation of D1 dopamine receptors in the SNR by dendritically released dopamine may facilitate locomotion by increasing GABA release in the SNR (Robertson and Robertson, 1988).

Enhanced GABA release from the terminals of the striatonigral fibers produces inhibition of the tonically active SNR neurons that project to the thalamus, superior colliculus, and pendunculopontine nucleus (Scheel-Kruger, 1986; Gauchy et al., 1987). Rotation thus depends on the activation (or disinhibition) of these motor nuclei as a result of increased activity of the striatonigral pathway (Scheel-Kruger, 1986). Consequently, D1 dopamine receptors in the SNR are strategically positioned for the gating of striatal outflow to motor nuclei (Savasta et al., 1986; Robertson and Robertson, 1987a, b). The ability of intranigral administration of haloperidol to reduce amphetamine-induced locomotion is consistent with the notion that 
dopaminergic receptor activation in the $\mathrm{SN}$ is central to the gating of striatal outflow (Jackson and Kelly, 1984).

Insufficient D1 dopamine receptor stimulation in the SNR, resulting from destruction of the SNC neurons, may, therefore, contribute to the akinetic syndrome characteristic of Parkinson's disease (Robertson and Robertson, 1987a, b). Recent studies using an animal model of Parkinson's disease suggest that the beneficial effect of L-dopa in Parkinson's disease may result from an elevation of dopamine content in the $\mathrm{SN}$ as well as the striatum (Robertson and Robertson, 1988). It has been reported that a poor relationship exists in this animal model between the content (Spencer and Wooten, 1984) or extracellular concentration of dopamine in the striatum and the duration of the behavioral effects of L-dopa (Zetterström et al., 1986). Our studies suggest that the elevation of dopamine content that occurs in the 6-OHDA-lesioned SN after peripheral L-dopa may persist longer and maintain locomotion after dopamine levels have declined in the ipsilateral striatum (Robertson and Robertson, 1988). Consequently, the following studies were undertaken to establish whether a temporal correlation exists between nigral dopamine content and the behavioral effects of L-dopa in an animal model of Parkinson's disease.

\section{Materials and Methods}

Animal preparation, drugs, and catecholamine determinations. Male Sprague-Dawley rats weighing 300-325 gm were anesthetized with sodium pentobarbital, and unilateral lesions were made by injection of $11.4 \mu \mathrm{g}$ of 6-hydroxydopamine (6-OHDA) $\mathrm{HBr}$ (Sigma)(to give $8 \mu \mathrm{g}$ 6-OHDA free base) in $4 \mu \mathrm{l}$ of sterile saline containing $0.5 \%$ ascorbic acid. The solution was injected, over $7 \mathrm{~min}$, into the rostral SN at the coordinates AP $-3.0, \mathrm{~L}$ (right) $1.8, \mathrm{~V}-7.8$, according to the atlas of Pellegrino et al. (1979). Behavioral screening was carried out after at least 2 weeks recovery, and animals that turned no less than 7 turns/ min to an apomorphine $(0.5 \mathrm{mg} / \mathrm{kg}$, i.p. $)$ challenge were included in the study. One week later, rats were randomly allocated to a vehicle $(0 \mathrm{hr})$ or 4 L-dopa treatment groups. On the experimental day, the 4 L-dopa groups received a carbidopa pretreatment $(25 \mathrm{mg} / \mathrm{kg}$, i.p.) followed 1 $\mathrm{hr}$ later by an $\mathrm{L}$-dopa injection $(25 \mathrm{mg} / \mathrm{kg}$, i.p.). The four L-dopa treatment groups differed only in the amount of time that elapsed between the L-dopa injection and death by cervical dislocation and decapitation. One of the $4 \mathrm{~L}$-dopa groups was killed at hourly intervals after L-dopa injection. The control $(0 \mathrm{hr})$ group received a carbidopa injection $(25$ $\mathrm{mg} / \mathrm{kg}$, i.p.), followed $1 \mathrm{hr}$ later by an injection of olive oil $(1 \mathrm{ml} / \mathrm{kg}$, i.p.). The control animals were killed immediately after the olive oil injection. After cervical dislocation and decapitation, the striatum and SN were dissected rapidly on ice. The striatal and nigral tissue samples were then homogenized in $0.1 \mathrm{~N}$ perchloric acid and stored at $-15^{\circ} \mathrm{C}$. Dopamine and 3,4-dihydroxyphenylacetic acid (DOPAC) levels were assessed by high-performance liquid chromatography (HPLC) with electrochemical detection, as described previously (Veinot et al., 1987). The limit of detection sensitivity was $62.5 \mathrm{pg}$. Dunnett's multiple $t$-test was used to compare the experimental dopamine concentration values to the respective $0 \mathrm{hr}$ control value. L-dopa (Merck, Sharp and Dohme) was suspended in olive oil $(25 \mathrm{mg} / \mathrm{ml}$ ), and SCH 23390 (Schering Plough Corporation) was dissolved in distilled water, $2 \mathrm{mg} / \mathrm{ml}$. Carbidopa (25 $\mathrm{mg} / \mathrm{ml}$ ) (Merck, Sharp and Dohme) was dissolved in warm sterile saline.

Chronic indwelling cannula surgery. In order to perform focal infusions of dopaminergic drugs in the striatum and the SNR, guide cannulas were implanted above these structures. Before surgery, the animal received a prophylactic intramuscular injection of antibiotic (chloracetin $30 \mathrm{mg}$, i.m.). C315G guide cannulas (Plastic Products Company, Roanoke, VA) were implanted so that the tip of the cannula terminated 3 $\mathrm{mm}$ from the center of the striatum or $5 \mathrm{~mm}$ from the center of the SNR. After a burr hole was made in the skull, the cannula was lowered into position and secured with dental acrylic. Guide cannulas were placed $3 \mathrm{~mm}$ dorsal to the center of the body of the striatum $(+0.5 \mathrm{AP}$, 3.2 ML right, $-3.0 \mathrm{DV})$ or $5 \mathrm{~mm}$ dorsal to the center of the SNR $(-5.2$ AP, 2.2 ML right, $-3.6 \mathrm{DV}$ ), using the flat skull orientation of Paxinos and Watson (1982). The wound was covered with polysporin to prevent infection, and the incision was closed with surgical suture (Ethicon). A
C313DC dummy cannula (Plastic Products) was then inserted into the guide cannula to prevent the entry of blood or foreign matter into the guide cannula. The animals were allowed a $7 \mathrm{~d}$ recovery period before further experimentation.

Focal infusion procedure. Cannulated animals were placed in a velcro harness and allowed to habituate to the rotometer environment for 30 $\mathrm{min}$ before the injection procedure began. The animals were then gently removed from the rotometer, the dummy cannula was removed, and a C3131 28-gauge injection cannula (Plastic Products) was inserted into the guide cannula and screwed into position. The animals were placed in a large (30 liter) plastic tub for the duration of injection. All injections were made at an infusion rate of $0.4 \mu \mathrm{l} / \mathrm{min}$, using a CMA 100 infusion pump (Carnegie Medicin, Stockholm, Sweden). In order to avoid nonspecific damage of the target tissue by the larger 26-gauge guide cannula, the injection cannula extended $3 \mathrm{~mm}$ and $5 \mathrm{~mm}$ beyond the tip of the striatal and SNR guide cannulas, respectively. At the end of the focal injection procedure, the injection cannula was left in place for an additional 90 seconds to permit diffusion of the drug to the surrounding area. The injection cannula was then removed, the dummy cannula was reinserted into the guide cannula, and the animal was returned to the rotometer. Injection volumes for the striatum were 1 or $3 \mu \mathrm{l}(2 \mu \mathrm{g}$ or 6 $\mu \mathrm{g} \mathrm{SCH} \mathrm{23390),} \mathrm{whereas} \mathrm{all} \mathrm{injections} \mathrm{in} \mathrm{the} \mathrm{SNR} \mathrm{were} \mathrm{in} \mathrm{a} 1 \mu \mathrm{l}(2 \mu \mathrm{g}$ $\mathrm{SCH} 23390$ ) volume. In the case of nigral focal infusions, animals were divided into 2 groups that received either $2 \mu \mathrm{g}$ of SCH 23390 in a $1 \mu \mathrm{l}$ volume or water alone into the SNR. Systematic injection of $L$-dopa $(25 \mathrm{mg} / \mathrm{kg}$, i.p.) followed $5 \mathrm{~min}$ later. Six days later, the 2 groups received the opposite treatments. All animals were pretreated with carbidopa $(25$ $\mathrm{mg} / \mathrm{kg}$, i.p.) $1 \mathrm{hr}$ before the peripheral L-dopa treatments. The number of rotations was determined at $10 \mathrm{~min}$ intervals for $4 \mathrm{hr}$ using an automated rotometer. Placement of the internal cannula was verified histologically. In the case of intrastriatal injections, half of the animals received the water injection, and the remaining animals received the SCH $23390(2 \mu \mathrm{g}$ in $1 \mu \mathrm{l})$ treatment on the first treatment day. One week later, the treatments were reversed, and 2 weeks after the first treatment, animals received the high dose of SCH $23390(6 \mu \mathrm{g}$ in $3 \mu \mathrm{l})$.

\section{Results}

\section{Striatal and nigral $D A$ and DOPAC content}

The peripheral L-dopa injection consistently induced circling in a direction contralateral to the side of the lesion in all the animals (over 150 rotations $/ 30 \mathrm{~min}$ ) for a $3.5 \mathrm{hr}$ period (Fig. $1 \mathrm{~A}$ ). As expected, a 6-OHDA lesion of the SN caused a $98 \%$ depletion of dopamine in the striatum and an $80 \%$ depletion of dopamine in the SN (Fig. 1, B, C). Following L-dopa, dopaminc levels increased in both the intact and the lesioned side in the striatum, but the relative increase was much greater on the lesioned side after L-dopa (Fig. $1 B$ ). The greater increase in dopamine on the lesioned side $(40 \%$ increase on the intact side compared with an $880 \%$ increase on the lesioned side) may reflect enhanced metabolic activity of the surviving dopaminergic neurons (Agid et al., 1973). DOPAC content in the intact striatum was increased significantly after L-dopa challenge and was elevated by more than 3-fold $2 \mathrm{hr}$ after the injection (Fig. $2 A$ ). In the denervated striatum, DOPAC content increased, relative to basal values, to a greater degree than DOPAC content in the intact striatum, i.e., 20-fold increase in DOPAC content $2 \mathrm{hr}$ after L-dopa (Fig. $2 A$ ). Consequently, the large increase in DA content in the denervated striatum, relative to the basal values $(0 \mathrm{hr})$, is paralleled by a pronounced increase in DOPAC content.

Although the L-dopa challenge only partially restored striatal dopamine levels (to about $10 \%$ of normal) (Fig. $1 B$ ), the same treatment was able to restore completely the dopamine content in the lesioned SN (Fig. 1C). DOPAC levels in the SN also mirror the complete recovery seen after L-dopa for dopamine levels (Fig. $2 B$ ). Interestingly, the L-dopa challenge significantly elevated dopamine and DOPAC concentrations in both the ipsilateral and contralateral $\mathrm{SN}$, although the relative increase in the lesioned SN was far greater. That is, L-dopa produced a 2.5- 
A.

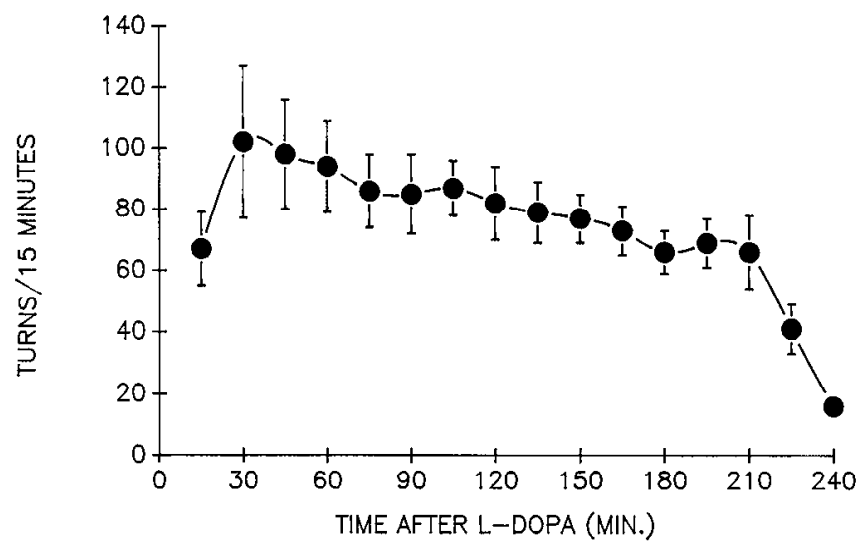

B.

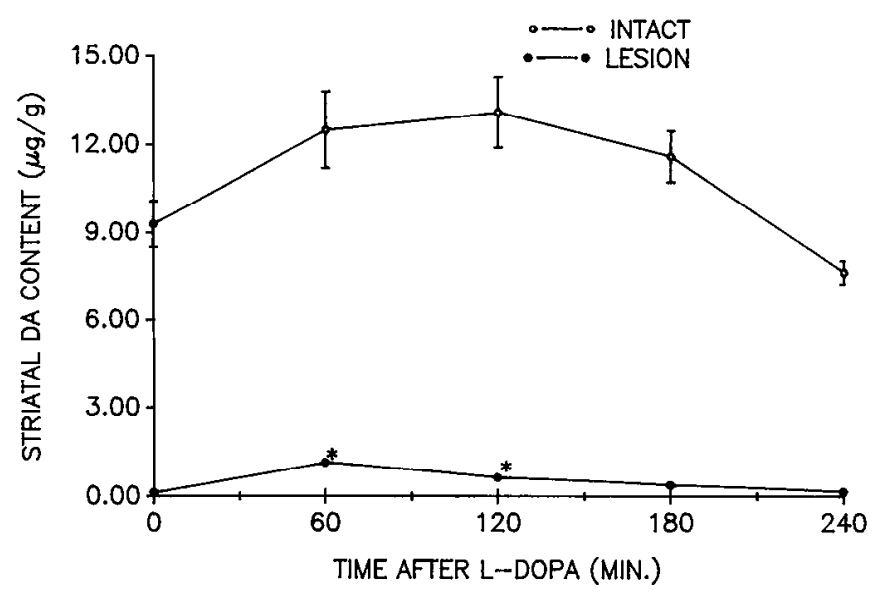

c.

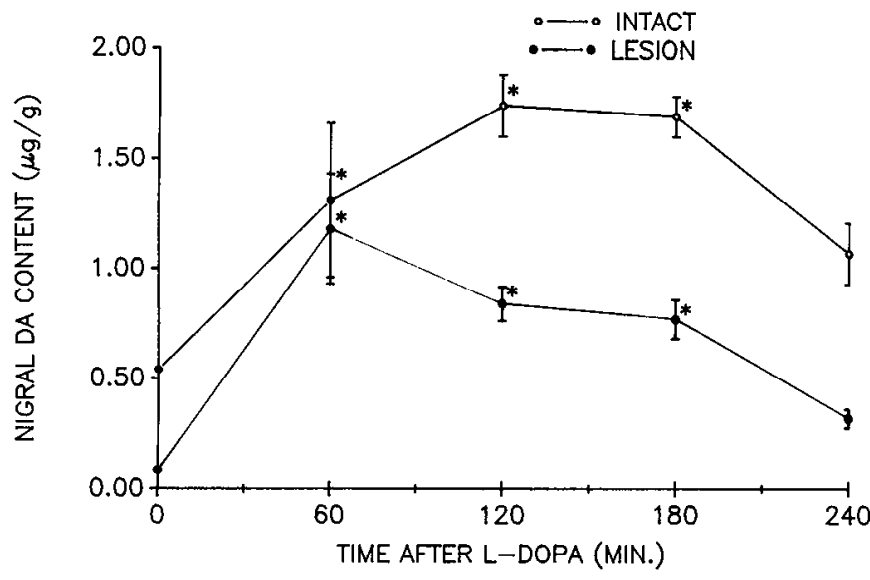

Figure 1. Effect of peripheral L-dopa $(25 \mathrm{mg} / \mathrm{kg}$, i.p.) challenge on rotational behavior and dopamine tissue concentration (wet weight) in the striatum and SN of the 6-OHDA lesioned rat. Each data point is the mean SEM for 4 animals. $A$, Rotation rates of 6-OHDA-lesioned rats measured at 15 min intervals for $4 \mathrm{hr}$. $B$, Dopamine tissue concentration in the intact and denervated striatum after vehicle or $L$-dopa in 6-OHDA-treated rats. $C$, Dopamine tissue concentration in the intact and 6-OHDA-lesioned SN after vehicle or L-dopa. Asterisk, $p<0.05$ for experimental groups relative to control $(0 \mathrm{hr})$. fold increase in dopamine content in the intact $\mathrm{SN}$, whereas dopamine concentrations were elevated 15 -fold in the 6-OHDAlesioned SN $1 \mathrm{hr}$ after injection (Fig. 1C). DOPAC content peaked in both the intact and lesioned SN 2 hr after L-dopa.

\section{Circling behavior}

Although the animals continue to rotate at a constant rate for $3.5 \mathrm{hr}$ after the L-dopa injection, at $2 \mathrm{hr}$ dopamine levels in the striatum have started to decline and, by $3 \mathrm{hr}$, are no longer significantly higher than control $(0 \mathrm{hr})$ values. Failure of the striatal concentration of dopamine to match temporally the pharmacological effects of L-dopa suggests that an additional site is responsible for the mediation of circling behavior. Dopamine levels remain elevated in the $\mathrm{SN}$ at $3 \mathrm{hr}$ and do not return to'control $(0 \mathrm{hr})$ values until the animals stop rotating at $4 \mathrm{hr}$. This suggests that elevated dopamine levels in the SN maintain circling behavior when striatal dopamine levels have begun to decline. To determine whether the persistent elevation of dopamine levels in the SN after L-dopa is of functional consequence for the 6-OHDA-lesioned animal, the selective D1 dopamine receptor antagonist SCH $23390(2 \mu \mathrm{g} / 1 \mu \mathrm{l})$ was infused directly into the SN 10 min before L-dopa challenge. Intranigral infusion of SCH 23390 produced a near complete blockade of circling behavior (Fig. 3), indicating that activation of $\mathrm{D} 1$ receptors in the $\mathrm{SN}$ is crucial not only to the maintenance but also to the full expression of $\mathrm{L}$-dopa-induced rotational behavior.

The effect of infusion of SCH 23390 into the ipsilateral striatum on L-dopa-induced rotational behavior is shown in Figure 4. In contrast to the SNR injections, injection of SCH 23390 (2 $\mu \mathrm{g}$ in $1 \mu \mathrm{l}$ of water) into the ipsilateral striatum did not reduce L-dopa-induced turning behavior (Fig. 4). However, infusion of $6 \mu \mathrm{g} \mathrm{SCH} 23390$ in $3 \mu \mathrm{l}$ of water produced a significant $(45 \%)$ reduction in L-dopa-induced turning behavior (Fig. 4).

\section{Discussion}

The major finding from the present studies is that L-dopa produces a dramatic increase in dopamine levels in the ipsilateral SN of 6-OHDA-lesioned rats that lasts for the full duration of rotational behavior. Consequently, there is a better correlation between the duration of turning behavior and the elevation of dopamine levels in the SN rather than between striatal dopamine levels and the duration of turning behavior. Moreover, the ability of intranigral administration of the selective D1 agonist SCH $23390(2 \mu \mathrm{g}$ in $1 \mu \mathrm{l})$ to block rotational behavior after L-dopa suggests that activation of D1 dopamine receptors in the SNR is, at lcast, nccessary for the maintenance of locomotion and may also be crucial for the initiation of turning behavior. This is in agreement with earlier studies in which we found that preventing the conversion of $\mathbf{L}$-dopa to dopamine with intranigral infusion of the dopa-decarboxylase inhibitor carbidopa blocks L-dopa-induced rotational behavior (Robertson and Robertson, 1988). Taken together, these studies suggest that $\mathrm{SCH} 23390$ attenuates rotational behavior by preventing newly synthesized and released dopamine from activating D1 dopamine receptors in the SNR. Since a 6-OHDA lesion of the SN eliminates most of the D2 dopamine receptors in the SN while sparing the high concentration of D1 dopamine receptors present on the striatonigral terminals, it seems likely that $\mathrm{SCH}$ 23390 is acting at Dr dopamine receptors (Savasta et al., 1986).

As expected, L-dopa administration elevated dopamine levels in the ipsilateral striatum (Fig. 1B). However, dopamine content 


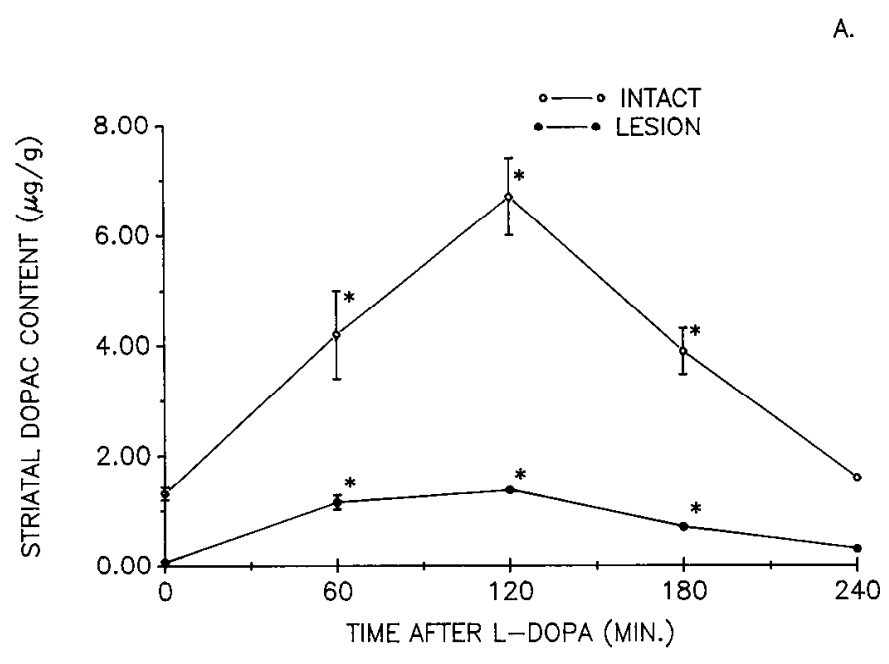

B.

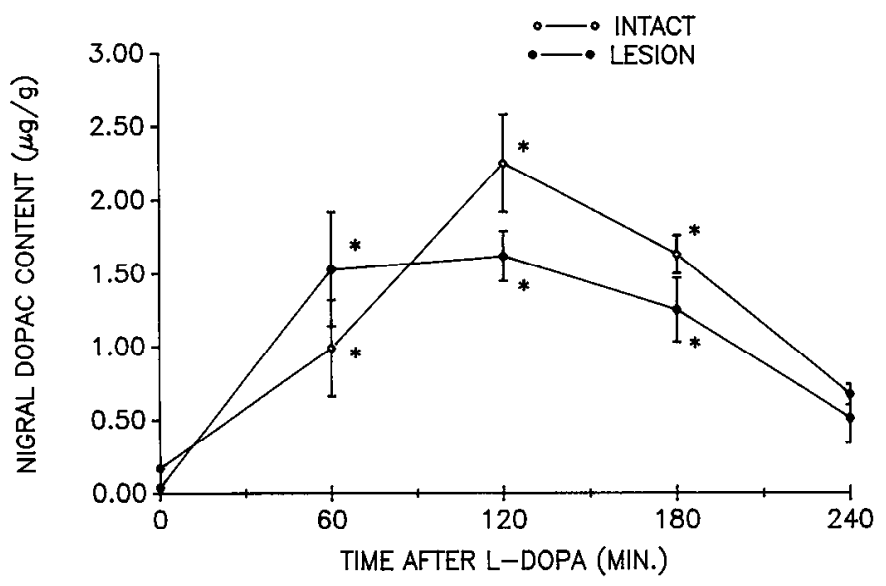

Figure 2. Effect of peripheral L-dopa ( $25 \mathrm{mg} / \mathrm{kg}$, i.p.) challenge on DOPAC tissue concentration (wet weight) in the striatum and $\mathrm{SN}$ of the 6-OHDA-lesioned rats. Each data point is the mean \pm SEM for 4 animals. $A$, DOPAC tissue concentration in the intact and denervated striatum after vehicle or L-dopa in 6-OHDA-treated rats. $B$, DOPAC tissuc concentration in the intact and 6-OHDA-lesioned SN after vehicle or L-dopa. Asterisk, $p<0.05$ for experimental groups relative to control $(0 \mathrm{hr})$.

in the ipsilateral striatum remained significantly elevated for only $2 \mathrm{hr}$ relative to the $0 \mathrm{hr}$ value. Intrastriatal administration of SCH $23390(6 \mu \mathrm{g} / 3 \mu \mathrm{l})$ significantly reduced L-dopa-induced rotational behavior, suggesting that activation of striatal D1 dopamine receptors may be related to the therapeutic action of L-dopa in Parkinson's disease. However, a high dose of SCH $23390(6 \mu \mathrm{g}$ in $3 \mu \mathrm{l})$ was needed to produce a significant but modest $45 \%$ reduction in L-dopa-induced rotational behavior. Apparently, some site (sites) in the brain is (are) capable of mediating circling behavior after intrastriatal SCH 23390 administration.

A 5-10 times greater reduction of dopamine levels in the SNR than the SNC in SN tissue from Parkinson patients has been reported (Bokobza et al., 1984). Interestingly, the levels of the dopamine metabolite homovanillic acid (HVA) were only slightly lower in the SNR than in the SNC, producing a ratio of HVA DA in the SNC of 22.2 and of 202 in the SNR (Bokobza et al., 1984). These results suggest significant metabolic compensation

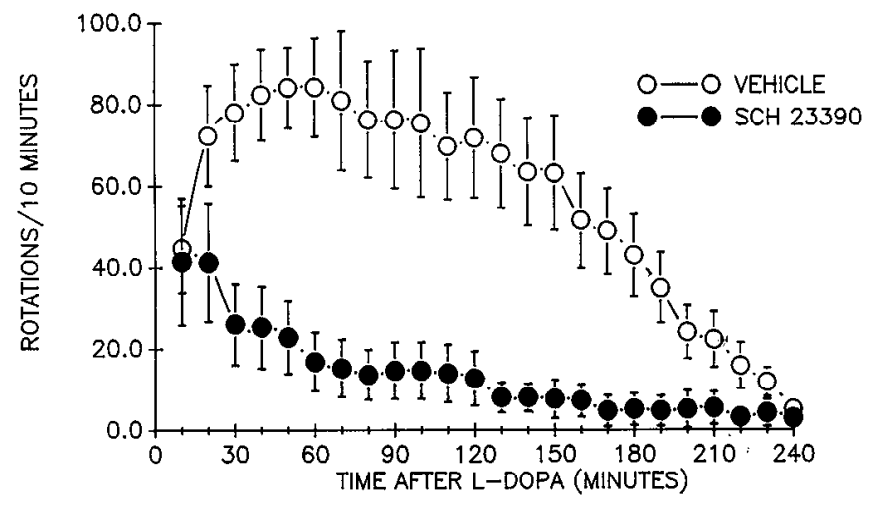

Figure 3. Comparison of the effect of intranigral injection of water (1 $\mu \mathrm{l})$ and SCH $23390(2 \mu \mathrm{g}$ in $1 \mu \mathrm{l})$ on the magnitude of turning after a peripheral L-dopa ( $25 \mathrm{mg} / \mathrm{kg}$, i.p.) challenge. Each data point is the mean \pm SEM of 9 animals.

in the SN, particularly the SNR, for the loss of dopaminergic neurons (Bokobza et al., 1984). Although, as these authors indicate, this is not a firm conclusion, it does support the findings of the present paper, which suggest that a 6-OHDA lesion of the SN induces significant metabolic compensation.

The present results indicate not only that the SN is a likely site for the action of L-dopa in Parkinson's disease but also that the therapeutic effect, at least in part, is mediated by $\mathrm{D} 1$ dopamine receptors in the SNR. In agreement with this notion, L-dopa and the DI agonist SKF 38393 have been found to increase dramatically the uptake of 2-deoxyglucose in the SNR of 6-OHDA-treated rats (Trugman and Wooten, 1986, 1987). The SNR contains almost exclusively dopamine receptors that are coupled to adenylate cyclase (D1 dopamine receptor) and are located presynaptically on the striatonigral pathway (Savasta et al., 1986). These D1 dopamine receptors are likely the target site for dopamine released from the dendrites of the SNC, which arborize in the SNR (Cheramy et al., 1981; Robertson and Robertson, 1987a, b). Consequently, L-dopa may exert its therapeutic action in the $\mathrm{SN}$ by restoring dopaminergic tone on D1 receptors located on the synaptic terminals of the striatonigral pathway (Savasta et al., 1986). In further support of this idea,

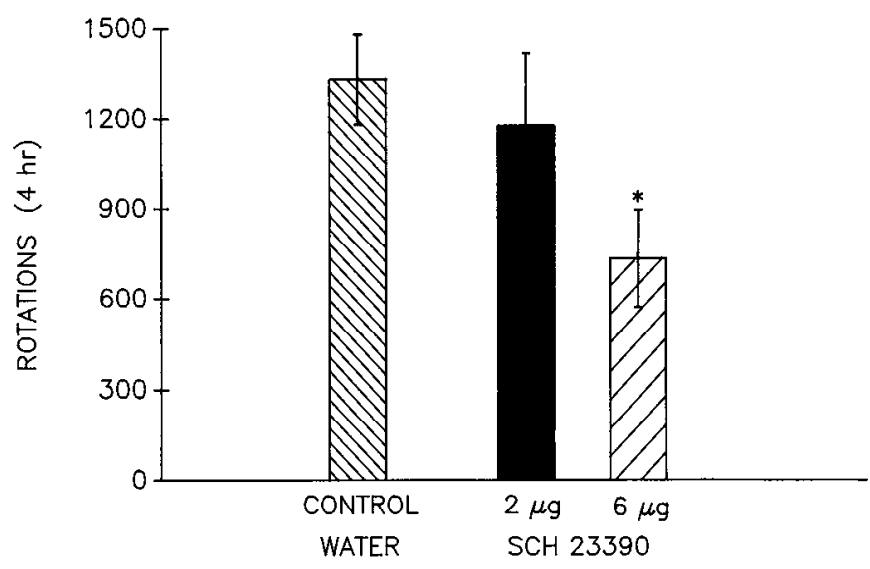

Figure 4. Effect of intrastriatal injection of water $(1 \mu \mathrm{l})$ and SCH 23390 ( $2 \mu \mathrm{g}$ in $1 \mu \mathrm{l}$ and $6 \mu \mathrm{g}$ in $3 \mu \mathrm{l}$ ) on the total number of turns completed in a $4 \mathrm{hr}$ period after a peripheral L-dopa challenge. Each data point is the mean $\pm \mathrm{SEM}$ of 12 animals. Asterisk, $p<0.05$ for experimental groups relative to control (water). 
Kozlowski et al. (1980) report that unilateral electrolytic lesions of the internal capsule markedly reduced the rate of contraversive turning produced by unilateral administration of apomorphine $(5 \mu \mathrm{g})$ into the SNR ipsilateral to these lesions in animals with a 6-OHDA lesion of the ipsilateral ventral mesencephalon. These authors argue that an electrolytic lesion of the internal capsule destroys the terminals of GABAergic fibers from the striatum and globus pallidus that innervate the SNR. Consequently, turning behavior after intranigral administration of apomorphinc is reduced because apomorphine can no longer activate the release of GABA from the terminals of these fibers.

The dopamine and DOPAC tissue content in the lesioned nigra at $1 \mathrm{hr}$ does not differ from the dopamine and DOPAC content of the intact nigra after L-dopa, suggesting that either the D1 dopamine receptors have become supersensitive to dopamine or that the SNR GABA receptors have become supersensitive to GABA released from the striatonigral pathway (Fig. $1 B$ ). The number of $\mathrm{D} 1$ receptors in the SNR, as measured by autoradiographic methods (Savasta et al., 1986), does not increase after a 6-OHDA lesion. However, enhanced sensitivity of dopamine-activated adenylate cyclase may occur in the absence of alterations in receptor number (Missale et al., 1987). Supersensitivity of the projection neurons to iontophoretically applied GABA in the SNR after chronic dopamine blockade has been demonstrated (Frey et al., 1987). Chronic haloperidol administration to rats for $30 \mathrm{~d}$ resulted in a significant reduction in the amount of iontophoretically applied GABA to inhibit the activity of neurons in the SNR (Frey et al., 1987). Homogenate binding revealed a significant $58 \%$ increase in the number of ${ }^{3} \mathrm{H}$-muscimol binding sites in the SNR from rats treated chronically with haloperidol (Frey et al., 1987). A comparable elevation of ${ }^{3} \mathrm{H}$-muscimol binding in the SNR has been demonstrated, using autoradiographic techniques, in rats with a unilateral 6-OHDA lesion of the ipsilateral medial forebrain bundle (Pan et al., 1985). Consequently, a reduction in dopamine receptor activation, resulting from destruction of the ascending dopaminergic neurons or chronic antagonist treatment, decreases the activity of striatal and pallidal efferents, producing an enhanced sensitivity of neurons in the SNR to GABA. Increased sensitivity of the SNR to GABA released from the terminals of striatal and pallidal fibers may, therefore, mediate the behavioral supersensitivity to intranigral administration of dopaminergic agonists after a 6-OHDA lesion of the SNC neurons. Thus, an increase in the number of GABA receptors rather than dopamine receptors in the SNR may mediate the behavioral effects of L-dopa. These findings demonstrate, as proposed earlier (Robertson and Robertson, 1987a, b) that the SN is an important site of action for L-dopa in an animal model for Parkinson's disease.

Our findings do not deprecate the role of the striatum but rather suggest that L-dopa alleviates the symptoms of Parkinson's disease by restoring dopaminergic tone not only in the striatum but in the $\mathrm{SN}$ as well. Our results suggest that focusing future attention on the role of the SN in Parkinson's disease will have implications for both the pathology and the therapy of this disorder.

\section{References}

Agid, Y., F. Javoy, and J. Glowinski (1973) Hyperactivity of remaining dopaminergic neurons after partial destruction of the nigro-striatal dopaminergic system in the rat. Nature New Biol. 245: 150-151.

Björklund, A., and O. Lindvall (1975) Dopamine in dendrites of the substantia nigra neurons: Suggestions for a role in dendritic terminals. Brain Res. 83: 531-537.

Bokobza, B., M. Ruberg, B. Scatton, F. Javoy-Agid, and Y. Agid (1984) $\left[{ }^{3} \mathrm{H}\right]$ Spiperone binding, dopamine and HVA concentrations in Parkinson's disease and supra nuclear palsy. Eur. J. Pharmacol. 99: 167175.

Bunney, B. S., and G. K. Aghajanian (1973) Comparison of effects of L-dopa, amphetamine and apomorphine on firing rate of rat dopaminergic neurons. Nature New Biol. 245: 123-125.

Cheramy, A., V. Leviel, and J. Glowinski (1981) Dendritic release of dopamine in the substantia nigra. Nature 289: 537-542.

Frey, J. M., M. K. Ticku, and R. D. Huffman (1987) GABAergic supersensitivity within the pars reticulata of the rat substantia nigra following chronic haloperidol administration. Brain Res. 425: 73-84. Gauchy, C., M. L. Kemel, R. Romo, J. Glowinski, and M. J. Besson (1987) The role of dopamine released from distal and proximal dendrites of nigrostriatal dopaminergic neurons in the control of GABA transmission in the thalamic nucleus ventralis medialis in the cat. Neuroscience 22: 935-946.

Geffen, L. B., T. M. Jessell, A. C. Cuello, and L. L. Iversen (1976) Release of dopamine from dendrites of rat substantia nigra. Nature 260: $258-260$.

Hornykiewicz, O. (1974) The mechanisms of action of 1-dopa in Parkinson's disease. Life Sci. 15: 1249-1259.

Jackson, E. A., and P. H. Kelly (1983) Role of nigral dopamine in amphetamine-induced locomotor activity. Brain Res. 278: 366-369.

Jackson, E. A., and P. H. Kelly (1984) Nigral dopaminergic mechanisms in drug-induced circling. Brain Res. Bull. 11: 605-611.

Kozlowski, M. R., S. Sawyer, and J. F. Marshall (1980) Behavioural effects and supersensitivity following nigral dopamine receptor stimulation. Nature 287: 52-54.

Missale, C., E. Nisoli, P. Liberini, P. Rizzonelli, N. Memo, M. Buonamici, A. Rossi, and P. Spano (1989) Repeated reserpine administration up-regulates the transduction mechanisms of D1 receptors without changing the density of $\left[{ }^{3} \mathrm{H}\right]-\mathrm{SCH} 23390$ binding. Brain Res. 483: $117-122$.

Nissbrandt, H., E. Pileblad, and A. Carlson (1985) Evidence for dopamine release and metabolism beyond the control of nerve impulses and dopamine receptors in the rat substantia nigra. J. Pharm. Pharmacol. 37: 884-889.

Pan, H. S., J. B. Penney, and A. B. Young (1985) $\delta$-Aminobutyric acid and benzodiazepine receptor changes induced by unilateral 6-hydroxydopamine lesions of the medial forebrain bundle. J. Neurochem. 45: 1396-1404.

Paxinos, G., and C. Watson (1982) The Rat Brain in Stereotaxic Coordinates, Academic, New York.

Pellegrino, L. J., A. J. Pellegrino, and A. J. Cushman (1979) A Stereotaxic Atlas of the Rat Brain, 2nd ed., Plenum, New York.

Reubi, J.-C., L. L. Iversen, and T. M. Jessell (1977) Dopamine selectively increases ${ }^{3} \mathrm{H}-\mathrm{GABA}$ release from slices of rat substantia nigra in vitro. Nature 268: 652-654.

Robertson, G. S., and H. A. Robertson (1987a) D1 and D2 dopamine agonist synergism: Separate sites of action. Trends Pharmacol. Sci. 8: 295-299.

Robertson, G. S., and H. A. Robertson (1988) Evidence that the substantia nigra is a site of action for L-dopa. Neurosci. Lett. 89: 204208.

Robertson, H. A., and G. S. Robertson (1987b) Combined L-dopa and bromocriptine therapy for Parkinson's disease: A proposed mechanism of action. Clin. Neuropharmacol. 10: 84-87.

Savasta, M., A. Dubois, J. Benavides, and B. Scatton (1986) Different neuronal location of $\left[{ }^{3} \mathrm{H}\right] \mathrm{SCH} 23390$ binding sites in pars reticulata and pars compacta of the substantia nigra in the rat. Neurosci. Lett. 72: 265-271.

Scheel-Kruger, J. (1986) Dopamine-GABA interactions: Evidence that GABA transmits, modulates and mediates dopaminergic functions in the basal ganglia and the limbic system. Acta Neurol. Scand. 73: $1-54$.

Spencer, S. E., and G. F. Wooten (1984) Pharmacologic effects of L-dopa are not closely linked temporally to striatal dopamine concentration. Neurology 4: 1609-1611.

Trugman, J. M., and G. F. Wooten (1986) The effects of L-dopa on the regional cerebral glucose utilization in rats with unilateral lesions on the substantia nigra. Brain Res. 79: 264-274.

Trugman, J. M., and G. F. Wooten (1987) Selective D1 and D2 do- 
pamine agonists differentially alter basal ganglia glucose utilization in rats with unilateral 6-hydroxydopamine substantia nigra lesions. J. Neurosci. 7: 2927-2935.

Veinot, J. P., M. R. Peterson, and H. A. Robertson (1987) Striatal dopamine after cortical injury: A reexamination. Exp. Neurol. 87 . 207-211.
Zetterström, T., M. Herrera-Marschitz, and U. Ungerstedt (1986) Simultaneous measurement of dopamine release and rotational behaviour in 6-hydroxydopamine denervated rats using intracerebral dialysis. Brain Res. 376: 1-7. 